\title{
Improving Lung Scintigraphy
}

$\mathbf{W}_{\text {i }}$ ography, the use of lung scintigrapl for the diagnosis of acute embolism has declined sharply. How ever, in selected patient groups, such as those with elevated serum creatinine or contrast allergies, lung scintigraphy continues to play an important role in the diagnosis of pulmonary embolism. As a result, work has continued on approaches to improve the accuracy of lung scintigraphy. Two articles in this issue, by Stein et al. (1) and Gutte et al. (2), highlight some of these advances.

Stein et al. note the sparseness of data on the accuracy of SPECT lung

\section{See pages 1987 and 1999}

scintigraphy with respect to objective reference tests. Although there is a growing impression that SPECT is more accurate than planar imaging, sufficient data to support this hypothesis are not available. The published studies generally involve small numbers of patients from a single institution. Furthermore, the methods used are not optimal. Often, SPECT is compared with planar imaging or CT angiography in terms of sensitivity and specificity, but it is known that different tests may merely move the observer's operating point - the tradeoff between sensitivity and specificity. When one is comparing 2 diagnostic tests, the receiver-operating-characteristic method is preferred, since it allows identification of the value of a test independent of a particular operating point. Further, good

Received May 28, 2009; revision accepted Jul. 2, 2009.

For correspondence or reprints contact: J. Anthony Parker, 330 Brookline Ave., Beth Israel Deaconess Medical Center, Harvard Medical School, Boston, MA 02215-5491.

E-mail: j.a.perker@ieee.org

COPYRIGHT (C) 2009 by the Society of Nuclear Medicine, Inc.

DOI: 10.2967/jnumed.109.065086 statistical methods exist for comparing tests using the area under the receiveroperating-characteristic curve.

There is a consensus that lung scan readings should be binary-positive or negative-sometimes with the inclusion of a "nondiagnostic" group. A few of us believe that some of our clinical colleagues have the ability to understand that lung scintigraphy has multiple outcomes, which can be more accurately described by likelihood ratios (3). Likelihood ratios have the additional advantage that they can be extended to the case of both multiple test outcomes and multiple disease states. In the case of pulmonary embolism, different disease states could be used to model the severity of disease. Irrespective of clinical implications, the tendency to bifurcate the data has lead to an oversimplification in the reporting of study results.

Several of the studies reviewed by Stein et al., and the study by Gutte et al., calculate sensitivities and specificities in subgroups that exclude patients with technically inadequate, intermediate, indeterminate, or nondiagnostic studies. Although it is common to report lung scan results in a subset of patients excluding patients with nondiagnostic tests (4), use of an intention-to-diagnose analysis (5) would offer advantages similar to those of the intention-to-treat analysis widely accepted for reporting therapeutic results.

The section on technical studies in the article by Stein et al. notes a large number of different SPECT V/Q imaging protocols that have been developed. Perhaps the most important technical issue is the ventilation agent. Xenon, which allows multiphase evaluation, is practical only for planar imaging. An ultrafine carbon suspension (Technegas; Cyclomedica) and ${ }^{81 \mathrm{~m}} \mathrm{Kr}$ have distributions more similar to ventilation than aerosols and may have a more stable distribution during SPECT data collection. However, the former is not available in some countries, such as the United States, and the latter is expensive. A second important technical issue that is just starting to be addressed is respiratory maneuvers during SPECT, including the use of respiratory gating.

The article by Gutte et al. is a prospective comparison of $\mathrm{CT}$ angiography with SPECT/CT V/Q scintigraphy making use of the low-dose CT component of SPECT/CT. The CT portion of SPECT/CT is another source of technical variability with regard to both breathing and radiation absorbed dose. The CT radiation absorbed dose is often divided into high-dose diagnostic CT and low-dose attenuationcorrection/anatomic-localization CT, and within each group many different doses have been used. Although not diagnostic, the attenuation-correction/ anatomic-localization $\mathrm{CT}$ provides considerable anatomic information, of which Gutte et al. make good use.

The study of Gutte et al. uses a composite reference standard including electrocardiography, ultrasound of the lower extremities, D-dimer levels, and clinical follow-up for $6 \mathrm{mo}$. The composite reference also includes side-by-side reading of the methods being studiedSPECT/CT V/Q scintigraphy and CT angiography. Use of side-by-side reading takes into account the relative positivity of each examination: in one patient the $\mathrm{CT}$ angiogram may provide strong evidence of embolization whereas the lung scan may be intermediate in outcome, and visa versa. Side-by-side reading also takes advantage of the fact that there may be a crosscorrelation between tests: one test may provide an explanation for the findings of another test. (A simple Bayesian combination of results does not include information from this cross-correlation.) In pulmonary embolism, for which the 
diagnosis often cannot be established definitively, a composite standard is often the best standard. However, it is important to keep in mind the possibility that some patients may be wrongly assigned to a disease category by the tests that are being studied, giving inflated accuracies, which may be different for the different tests.

The study of Gutte et al. is not inconsistent with the widely held impression that anatomic information increases accuracy when added to V/Q imaging. Of 196 patients screened, only 100 patients were eligible. There was no final diagnosis in 5, CT angiography was technically inadequate in 6 , and V/Q imaging was of poor technical quality in 8 , leaving 81 patients. The point estimates of sensitivity and specificity for V/Q SPECT in a subset of 77 patients were $97 \%$ and $88 \%$. Including information from the low-dose CT in a subset of 81 patients, these estimates were high, $97 \%$ and $100 \%$. Furthermore, 4 patients with nondiagnostic V/Q scans were considered diagnostic using the anatomic CT information. V/Q SPECT with chest radiography was not compared with these results.

Gutte et al. also found better point estimates in a subset of patients using V/Q imaging and low-dose CT $(n=81)$ than in a subset using perfusion imaging and low-dose CT $(n=69)$ (sensitivity, $97 \%$ vs. $93 \%$; specificity, $100 \%$ vs. $51 \%$ ). This result is compatible with the traditional impression that ventilation imaging improves accuracy when added to perfusion and anatomic imaging. However, this result appears to be at variance with the results using 2 methods not including the ventilation scan, the Prospective Investigative Study of Acute Pulmonary Embolism Diagnosis (PISA-PED) and modified Prospective Investigation of Pulmonary Embolism Diagnosis (PIOPED) II criteria. When the PISA-PED and modified PIOPED II criteria were compared with the original PIOPED II results on the PIOPED II database, the point estimates of sensitivity and specificity were similar: sensitivity of $80.5 \%$ and $84.9 \%$, compared with $77.4 \%$, and specificity of $96.6 \%$ and $92.7 \%$, compared with $97.7 \%$, with fewer nondiagnostic studies $(0.0 \%$ and $20.6 \%$, compared with $26.5 \%$ ) (4).

Notably, 48 patients of the 96 patients excluded from the study by Gutte et al. were excluded because of contraindications to contrast. Furthermore, the CT angiography point estimate of sensitivity, 68\% ( $n=81)$, was lower than that for SPECT/CT V/Q imaging incorporating the low-dose CT information, 97\% $(n=81)$; both studies had point estimates of specificity of $100 \%$. These results are not inconsistent with the hypothesis that SPECT/CT V/Q imaging using ${ }^{81 \mathrm{~m}} \mathrm{Kr}$ is equivalent in accuracy to CT angiography.

Diagnosis of venous thromboembolism remains a difficult problem. Many people die with undiagnosed pulmonary embolism ( 6$)$, and there is concern that pulmonary embolism may be overdiagnosed in other patients (7). Venous thromboembolism represents a spectrum of disease. Small pulmonary emboli on CT angiography without apparent deep venous thrombosis on ultrasound may not need therapy (8), whereas massive emboli with hemodynamic compromise may require aggressive therapy (9). The role of lung scintigraphy in selection of therapy is not well defined. The extent of perfusion defects on planar lung scintigraphy correlates with cardiac ultrasound evidence of right ventricular hypokinesis, a physiologic marker of hemodynamic compromise (10). Evaluation of the severity of embolization has not been a recent focus of lung scan investigation. Because SPECT may provide a more accurate measure of severity than planar lung scintigraphy, the time may be appropriate to revisit the utility of lung scintigraphy for evaluating the severity of embolization.

The articles by Stein et al. and Gutte et al. suggest that SPECT or SPECT/CT V/Q imaging may considerably improve the diagnosis of pulmonary embolism, compared with planar imaging. However, these articles also raise several questions. Is SPECT truly superior to planar imaging? Which ventilation agent should be used? Is the anatomic information from low-dose CT superior to that from chest radiography? What CT radiation absorbed dose is acceptable? Should respiratory maneuvers or gating be used? Does ventilatory imaging add to perfusion plus anatomic imaging? How does optimized lung scintigraphy compare with CT angiography in a multiinstitutional setting? Although it is unlikely that the effect of these factors on diagnostic accuracy will be rigorously evaluated, this uncertainty is a problem we always face in diagnostic imaging.

For those of us whose goal it is to perform evidence-informed, practicebased medicine, these articles suggest that SPECT V/Q scintigraphy, or SPECT/CT V/Q scintigraphy making use of the information from CT, are worth evaluating in our own practices.

\section{J. Anthony Parker}

Beth Israel Deaconess Medical Center

Harvard Medical School

Boston, Massachusetts

\section{REFERENCES}

1. Stein PD, Freeman LM, Sostman HD, et al SPECT in acute pulmonary embolism. J Nucl Med. 2009;50:1999-2007.

2. Gutte H, Mortensen J, Jensen CV, et al. Detection of pulmonary embolism with combined ventilationperfusion SPECT and low-dose CT: head-to-head comparison with multidetector CT angiography. $J$ Nucl Med. 2009;50:1987-1992.

3. Parker JA, Coleman RE, Siegel BA, Sostman HD, McKusick KA, Royal HD. Procedure guideline for lung scintigraphy: 1.0. J Nucl Med. 1996;37:19061910.

4. Sostman HD, Miniati M, Gottschalk A, Matta F, Stein PD, Pistolesi M. Sensitivity and specificity of perfusion scintigraphy combined with chest radiography for acute pulmonary embolism in PIOPED II. J Nucl Med. 2008;49:1741-1748.

5. Knottnerus JA, Muris JW. Assessment of the accuracy of diagnostic tests: the cross-sectional study. J Clin Epidemiol. 2003;56:1118-1128.

6. Pineda LA, Hathwar VS, Grant BJB. Clinical suspicion of fatal pulmonary embolism. Chest. 2001;120:791-795.

7. Glassroth J. Imaging of pulmonary embolism: too much of a good thing? JAMA. 2007;298:2788-2789.

8. Anderson DR, Kahn SR, Rodger MA, et al. Computed tomographic pulmonary angiography vs ventilation-perfusion lung scanning in patients with suspected pulmonary embolism: a randomized controlled trial. JAMA. 2007;298:2743-2753.

9. Goldhaber SZ. Pulmonary embolism. Lancet. 2004;363:1295-1305

10. Wolfe MW, Lee TR, Feldstein ML, Parker JA, Come PC, Goldhaber SZ. Prognostic significance of right ventricular hypokinesis and perfusion lung scan defects in pulmonary embolism. Am Heart J. 1994;127:1371-1375. 